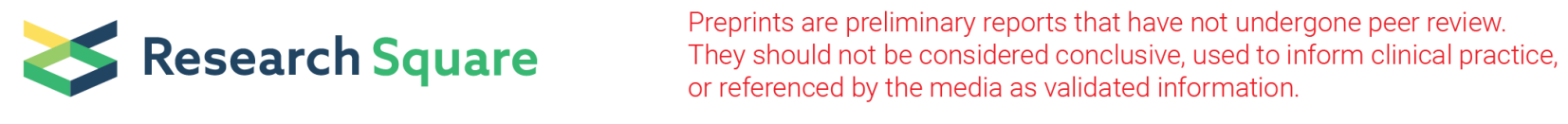

\title{
Sex difference in infant mortality: a study of UK Paediatric Intensive Care admissions over 11 years
}

Ofran Almossawi ( $\sim$ ofran.almossawi@nhs.net)

UCL Great Ormond Street Institute of Child Health https://orcid.org/0000-0001-6900-3590

Scott O'Brien

Imperial College Healthcare Trust

Roger Parslow

University of Leeds

Simon Nadel

Imperial College Healthcare Trust

Luigi Palla

London School of Hygiene \& Tropical Medicine https://orcid.org/0000-0001-6944-1750

Article

Keywords: infant mortality, paediatric intensive care unit, sex difference

Posted Date: October 9th, 2020

DOI: https://doi.org/10.21203/rs.3.rs-86410/v1

License: (c) (i) This work is licensed under a Creative Commons Attribution 4.0 International License. Read Full License 


\section{Abstract}

Rationale Within the UK, child mortality from all causes has declined for all ages over the last three decades. However, distinct inequality remains, as child mortality rates vary by sex and are generally found to be higher in males. A significant proportion of childhood deaths in the UK occur in Paediatric Intensive Care Units (PICU). Objectives We aimed to study the association of sex with infant mortality in PICUs. Methods We obtained data for all infant admissions to UK-PICUs from 01/01/2005 to 31/12/2015. We assessed the causal relationship between sex and mortality and selected appropriate variables for adjustment. We fitted a cause specific hazard ratio (CSHR) model, and a logistic model to estimate the adjusted effect of sex on mortality in PICU. Pre-defined subgroups were children less than 56 days old, and those with a primary diagnosis of infection. Measurements and Main Results Within the 71,243 admissions, there were 1,411/29,520 (4.8\%) female deaths, and 1,809/41,723 (4.3\%) male deaths. The adjusted male to female CSHR was 0.87 (95\%-Cl 0.81 to 0.92$)$ representing a $13 \%$ higher risk of death for females over males. Similarly, the adjusted OR for male to female mortality is 0.87 (95\%-Cl, 0.81 to 0.94$)$. Subgroup analysis yielded similar findings. Conclusions Female sex is associated with higher mortality in infants needing PICU admission, despite similar severity of illness to males. There was no effect of age or infection status. This suggests that females and males differ in the pathophysiology of critical illness and/or their response to treatment.

\section{At A Glance}

Scientific Knowledge on the Subject

Few studies have quantified any differential mortality of infants in paediatric intensive care units (PICUs), or have directly addressed the relationship between sex and mortality as a research aim. Other studies which indirectly examined mortality and sex all show very similar results in favour of better male survival in PICU, which is in the opposite direction of the estimates in the general population.

Added value of this study

Our multi-centre study is the first and largest to directly address the primary question of the relationship between sex and mortality in infants admitted to PICU. We justified our adjustment variables using causal graphs. We found that females had a $13 \%$ higher adjusted risk of mortality over males. We achieved similar results for subgroups of infants; before and after the time of primary infant vaccination, and in those with infection as the primary reason for admission. The mortality risk in UK PICUs has declined steadily over the study period for both sexes, but remains higher for females. Our results raise important questions regarding unmeasured environmental and/or biological factors which influence sex inequalities in infants for both admission and mortality in PICUs.

Twitter content

The largest study addressing the effect of sex on PICU-mortality in infants using UK-wide data: females had $13 \%$ higher risk of mortality

\section{Background And Introduction}

Child and infant mortality are important indicators of child health. The September 2010 United Nations Summit set a target for the fourth millennium development goal (MDG) to reduce the mortality rate of children under five years of age by two thirds between 1990 and $2015^{1}$. Over the past three decades, there have been sizeable reductions in infant and child mortality throughout resource rich countries mainly because of improvements in obstetric, paediatric medical and surgical care, and positive public health initiatives. Within the UK alone, child mortality from all causes has declined over all ages by $50-70 \%$ between 1980 and $2010^{2,3}$. Despite this progress, distinct inequality remains, as child mortality rates vary by sex and are generally found to be higher in males ${ }^{4}$. The 2018 mortality rate per 100,000 for England and Wales show male-female mortality ratios of 1.21 at age under 1 year, 1.26 at age $1-4$ years, 1.00 at age $5-9$ years, and 1.57 at age $10-14$ years ${ }^{5}$. A similar survival advantage for females is also observed in extremely premature neonates ${ }^{6}$ and historically has been hypothesized to be linked to a sex ratio at birth more in favour of males ${ }^{7}$.

Causes of child and infant mortality are complex and include biological, environmental, and behavioural influences. In the child and adolescent age group, the sex mortality difference is thought to be mainly related to external factors such as environmental and behavioural influences. The authors of a retrospective analysis of neonatal outcomes over eight years in New South Wales in Australia, found that incidence of grade III/IV

intraventricular haemorrhage and sepsis was higher in male neonates ${ }^{8}$. Overall mortality was also significantly higher for male neonates. In the UK, childhood mortality is relatively low, with infant (0-12 months) death rate at 3.6 per 1,000 live births. Death from infectious diseases in children (0-4 years) is 63.9 per $100,000^{9}$. 
Over $65 \%$ of childhood deaths in the UK occur in hospitals ${ }^{10}$, and over $85 \%$ of these deaths occur in Paediatric Intensive Care Units (PICUs) ${ }^{11}$. With respect to the specific effects of infections in childhood, it has been suggested that the two sexes may have variable immune responses to infections as mediated by their sex-hormones ${ }^{12}$.

Preliminary analyses of admissions of infants to UK PICUs due to infections reported larger numbers of male admissions over a two year period, but a greater female than male infant mortality ${ }^{13}$. A follow up to this analysis was carried out over a five-year period and produced similar results ${ }^{14}$.

Aims

We designed the present study with the primary aim to investigate the difference in "mortality within PICU" between male and female infants admitted to PICUs in the UK. A secondary aim was to clarify the conditions and data required for estimating the causal effect of sex on mortality.

\section{Methods}

\section{Data source}

After gaining ethical approval (London School of Hygiene \& Tropical Medicine, MSc Ethics Ref: 10684) we obtained data for all infant admissions to UK PICUs from the Paediatric Intensive Care Audit Network (PICANet) from $1^{\text {st }}$ January 2005 to $31^{\text {st }}$ December 2015 . PICANet is a national audit database recording the demographic and clinical data of children admitted to UK PICUs. The Collection of personally identifiable data by PICANet has ethical approval and has been approved by the Health Research Authority Confidentiality Advisory Group. The data is collected by individual PICUs as daily activity data, or Paediatric Critical Care Minimum Data Set (PCCMDS) ${ }^{15}$. The data contains anonymised information on individual patients from all 35 UK PICUs. Also linked to this dataset was the Index of Multiple Deprivation (IMD) score ${ }^{16,17}$, a UK government qualitative measure of deprived areas.

\section{Description of variables}

The relevant variables available in this dataset were: age (in days, converted to 30 day intervals); primary diagnosis of infection; deprivation score (IMD) 2010) ${ }^{17}$; PIM2R score (Paediatric Index of Mortality-2 Recalibrated, 2016 recalibration) which is a paediatric risk of mortality score calculated on admission to PICU 18; whether the patient received invasive ventilation and duration of invasive ventilation (in days); inotropic support; renal support; planned versus unplanned admission; gestational age (in weeks); and ethnicity (four categories). From primary diagnosis of infection, we selected out primary diagnosis of bronchiolitis, as this diagnosis is known to have a significantly lower mortality risk than other infections ${ }^{19,20}$.

Statistical methods

\section{Descriptive statistics}

For descriptive analysis, we used proportions and ratios to summarise categorical data, and means, standard deviations for numerical data or medians and first/third quartiles for very skewed distributions of numerical variables. As a descriptive summary of mortality, we fitted an unadjusted and PIM2R-adjusted logistic model for each PICU to describe the variability of the outcome between PICUs. To estimate the overall PIM2R-adjusted odds ratio, we fitted a logistic model with cluster robust standard errors (to account for clustering by PICU). We removed children that were still in PICU after 100 days $(n=192)$ as these were considered to have an atypical Length of Stay (LOS). The median LOS for a PICU stay in infants up to one-year of age (in the period 2014 to 2016) was 4.8 days (PICANet 2017 report). As the dataset for our study spans 11 years over which medical techniques in paediatric critical care have advanced, we generated a time-series plot to describe the mortality trends.

Causal analysis and causal diagrams

We drew directed acyclic graphs (DAGs) that represented the hypothesized causal relationships between the variables (see Supplementary materials) and showed under which scenarios we could derive valid estimates (these are detailed in the appendix). In summary, the causal diagram 
assumptions we made (DAG C in Supplementary) indicated that, in order to obtain a causal estimate of the direct effect of sex on mortality in PICU, we had to adjust our statistical models for the variable PIM2R.

Time-to-event analysis

Death within PICU and discharge from PICU (either to home or to another hospital) are two mutually exclusive competing risks. As the dataset had very little censoring we used a logistic regression model for PICU mortality at 100 days as the main analysis, and additionally used a cause specific hazard model CSHR) censored at 100 days to investigate possible time trends within those 100 days.

For the CSHR, children were censored at discharge. We assessed the assumption of proportionality of hazard (time varying coefficients), after fitting a Cox proportional hazard model including the selected adjustment variables, by plotting the Schoenfeld residuals for all the variables in the model. We carried out further assessments of proportionality using parametric restricted cubic splines, to better characterise the variability of the hazard ratios over time for all the variables in the model, via the Stata post estimation command STPHCOXRCS ${ }^{21}$. We used clinical justifications to decide on the placement of the internal knots at 2, 5, and 20 days. We fitted the final CSHR model using the adjustment variables selected though the DAG analysis. We included an interaction of splines with time for the variables sex and PIM2R, each with three internal knots placed at (2, 5 and 10 days). We assessed the functional forms of continuous variable (PIM2R) using Martingale residuals. All steps above were carried out using cluster robust standard errors to account for unobserved variability between PICUs.

For logistic regression analysis we assessed the functional forms of the continuous variables using lowess plots. We then fitted a logistic model including death, sex and any other adjustment variables. Length of stay in PICU is incorporated in the model by using time-to-event analysis, but otherwise we did not include this as an adjustment variable in the logistic model.

Subgroup analyses

Our predefined subgroup analyses were to test the hypotheses that sex mortality differs within the subgroups of children with a primary diagnosis of infection, and in children under 56 days (at PICU admission), which was presumed to be before their first routine infant vaccination.

To identify patients with a primary diagnosis of infection, ICD10 codes were used. Infants admitted with a diagnosis of bronchiolitis were not included in the infections category as stated above.

\section{Results}

After data preparation, 71,243 infants were included in the final analysis. Figure 1 summarises the characteristics of the excluded individuals. Observations where the sex or outcome were missing were removed. We excluded patients with a PICU LOS of less than two hours who were discharged alive, as these were not considered to be true PICU stays ${ }^{2}$. For children with multiple PICU admissions, only the first admission was retained in the dataset to reduce any bias resulting from correlated data (since multiple admissions are indicated but not linked in the dataset). And finally, observations with missing LOS data were removed due to lack of time to event information. Of the 71,243 admissions, 43,488 (61\%) had LOS of four days or less and out of 3,220 deaths, 1,677 (52\%) occurred in the first four days.

The overall summary of baseline characteristics is presented in Table 1. Out of all PICU admissions $58.6 \%$ were males. The distributions of clinical characteristics were very similar for the two sexes.

There were 1,411/29,520 (4.8\%) female deaths, and 1,809/41,723 (4.3\%) male deaths. There were numerically more male deaths than female deaths because more males were admitted than females. However, as Figure 2 shows, the proportion of females who died was consistently higher than males in every year, over the 11 year study period. 
The gap of crude percentage mortality within each sex has narrowed over the last decade. Both sexes show a reduction in percentage mortality over the years, with females experiencing a sharper decline than males. The Crude and PIM2R adjusted odds ratios for mortality, per PICU and overall, are presented in Figure 3.

The final variables we included in the time-to-event analysis and logistic regression were sex and PIM2R (converted to percentage risk of death). After running a Cox proportional hazard ( $\mathrm{CPH})$ model, the Schoenfeld residual plots and the flexible parametric post-hoc diagnostic plots showed a proportional hazard ratio for sex, and a time varying hazard ratio for PIM2R. The male to female CSHR was 0.87 ( $95 \% \mathrm{Cl} 0.81$ to 0.92$)$ representing a $13 \%$ higher risk of death for females over males.

The final CSHR model, using splines (to model the non-proportional effects of PIM2R), and cluster robust standard errors, is presented in Table S1 of the supplementary materials. Table 2 shows the cumulative proportions of deaths and discharges at various intervals for the first 40 days of PICU admission. Female deaths are consistently higher than male deaths at each point.

The parameters in Table 3 are from the logistic regression model with cluster robust standard errors to account for variations between the 35 PICUs. There is strong evidence for $14 \%$ lower odds of mortality for males compared to females. The OR of male to female deaths in Table 3 supports the conclusion derived from the time to event analysis (the CSHR).

The subgroup analyses for logistic regression (Tables S2 to S5 in the Supplementary materials) estimated a male to female OR of 0.84 (Cl= 0.75 to $0.94)$ for children $<56$ days of age and of $0.88(\mathrm{Cl}=0.80$ to 0.97$)$ for children $>56$ days; for the interaction effect between age subgroups and sex $\mathrm{p}=0.48(\mathrm{Cl}=0.81$ to 1.10$)$. The OR was $0.87(\mathrm{Cl}=0.73$ to 1.04$)$ for children with primary diagnosis of infection and of $0.85(\mathrm{Cl}=0.77$ to 0.93$)$ for children with primary diagnosis other than infection (but including bronchiolitis); for the interaction effect between infection subgroup and sex $\mathrm{p}=0.92(\mathrm{Cl}=0.80$ to 1.28$)$. The results of time to event analysis (HR) were very similar (see Tables S6 to S9 in the Supplementary materials).

\section{Discussion}

It is well described that there are more male than female deaths in most analyses of mortality data in infants and children. However several recent studies have suggested greater female than male mortality in intensive care ${ }^{22-25}$. Given that a sizable proportion of childhood deaths in the UK occur in PICU, we deemed PICU admissions a relevant cohort in which to examine potential sex differences in mortality. Our study differs from previous published literature in that it is the largest study in which the primary aim is to address the sex mortality difference in PICU, with careful consideration to the causal relationships between common variables and their relationship to the outcome. In particular, by drawing causal graphs connecting measured and unmeasured relevant variables, we clarified the data and conditions required to draw causal estimates of the direct effect of sex on mortality in PICU and highlighted the limitations of the current analysis.

The main finding from our analysis is that female sex is associated with higher mortality in PICU for infants up to 12 months of age. This finding is supported by strong evidence from both analysis methods we have used. There was no evidence of effect modification by the subgroups of infants under/over the age of 56 days, thus suggesting no clear effect of primary immunisation, or with a primary diagnosis of infection. When compared to the relative mortality in the general population where male infants are reported to have higher mortality, both the CSHR and the OR are in the opposite direction. Higher odds/hazard of mortality for female infants in PICU persisted across all subgroups of infants.

Over the 11-year duration of the study we observed a decline in PICU mortality for both sexes, but with a sharper decline for females. However, the sex mortality difference between females and males that we have described remained.

Using simple probability calculations, the higher number of male admissions to PICUs, together with the lower rate of male infant deaths in PICU leads to posterior odds of 0.79 when assessing PICU deaths. This is still overall in favour of female survival and consistent with the mortality rate ratio calculated in the overall population of 0.82 based on published national statistics figures (ONS death summary tables of 2016 , https://www.ons.gov.uk/) for under one year of age female to male mortality per 1000 live births. Hence the data we report do not contradict the 
numerically greater overall mortality of males (see calculations of posterior probabilities in supplementary materials). Rather, it warrants further investigation into the reasons for the greater proportion of males admitted and the greater mortality of females in PICUs.

Our results are in line with four other studies, two of which indirectly investigated the effect of sex on mortality and others observing this effect as a secondary finding, in children with varying age ranges. These studies were carried out in the United Sates, $2011^{22}$, Spain, $2015^{23}$, Sweden, $2017^{24}$, and the UK ${ }^{25}$. The US study had a large sample size of over 80,000 cases which included children up to 18 years. It was a multi-centre study with over 31 PICUs and spanning 2005-2008. The focus of that study was to assess if ethnicity had an effect on PICU mortality and the effect of being female, which was only used as an adjustment confounder, was estimated as $12 \%$ higher odds of PICU mortality over males ( $p=0.019){ }^{22}$. The Swedish study analysed data for 21,972 children over an eight year period (2008-2015), and included all PICU admissions in Sweden < 16 years. They reported a sex difference in the HR of mortality of 0.91 for boys, $p=0.035^{24}$. The Spanish single centre study of children $0-18$ years admitted to PICU over a period of three years showed an in-hospital mortality advantage of males over females $(3.3 \% \text { versus } 4.9 \%, p=0.042)^{23}$. The UK study, with a sample size of 154,667 and aged $<18$ years, investigated mortality in PICU for children with and without life-limiting conditions. Sex was used as an adjustment variable with a mortality odds ratio $1.09(p=0.002)$ for females compared to males ${ }^{25}$.

All these studies were conducted in high-resource settings with access to advanced critical care facilities for children. In these studies, gender was primarily used as an adjustment variable. Our current study confirms that the higher PICU mortality rate for females is consistent with that previously described over multiple healthcare settings i.e. socialised systems and private healthcare facilities. Strengths of our study are that it directly addresses the impact of sex on mortality and infants and it spans a longer duration than the other studies, with a primary focus on sex differences in mortality.

It is unclear what is driving the greater mortality risk for female infants in PICU. One hypothesis is that there are biological differences in the response to critical illness or in the response to interventions. Another implication from our study is that the quantification of severity of illness using PIM2R, which is calculated within the first hour of admission to PICU and is calculated independent of sex, should be recalibrated to account for sex. Since the PIM2R score had similar distributions between male and female infants, it is possible that female infants have a different trajectory of illness. Males and females may present with the same PIM2R score but at different stages of their illness, with males admitted to PICU earlier in the course of their illness.

Limitations

Our study does not address any potential explanations for the observed sex difference in mortality as we did not have access to data factors that may influence PICU admission, such as maternal or birth factors.

PICANet data collection is carried out by individual PICUs and thus may vary in data collection. However, data collection is closely audited and sex and outcome are expected to be consistently recorded.

In addition, data is censored beyond discharge so it is not possible to assess if the mortality difference continues beyond discharge from PICU.

\section{Conclusions}

We have shown strong evidence of greater relative mortality for female than male infants in PICUs across the UK. This persists after subgrouping by infection diagnosis and age prior to routine infant vaccination. This suggests that females and males differ in the pathophysiology of critical illness and/or their response to treatment.

The reasons for the disparity in outcome between females and males remains unclear and warrants further investigation in this and other populations.

\section{Declarations}

Declaration of interests 
We declare no competing interests.

\section{References}

1. United Nations General Assembly. MDG 4. http://www.un.org/millenniumgoals/pdf/MDG_FS_4_EN.pdf (2015).

2. O'Brien, S., Nadel, S., Almossawi, O. \& Inwald, D. P. The Impact of Chronic Health Conditions on Length of Stay and Mortality in a General PICU. Pediatr. Crit. Care Med. J. Soc. Crit. Care Med. World Fed. Pediatr. Intensive Crit. Care Soc. 18, 1-7 (2017).

3. Hardelid, P. et al. Estimating the prevalence of chronic conditions in children who die in England, Scotland and Wales: a data linkage cohort study. BMJ Open 4, e005331 (2014).

4. Sidebotham, P. et al. Understanding why children die in high-income countries. The Lancet 384, 915-927 (2014).

5. Office for National Statistics. Deaths broken down by age, sex, area and cause of death. https://www.ons.gov.uk/peoplepopulationandcommunity/birthsdeathsandmarriages/deaths\#publications.

6. Peacock, J. L., Marston, L., Marlow, N., Calvert, S. A. \& Greenough, A. Neonatal and infant outcome in boys and girls born very prematurely. Pediatr. Res. 71, 305-310 (2012).

7. Pongou, R. Why is infant mortality higher in boys than in girls? A new hypothesis based on preconception environment and evidence from a large sample of twins. Demography 50, 421-444 (2013).

8. Kent, A. L., Wright, I. M. R., Abdel-Latif, M. E. \& New South Wales and Australian Capital Territory Neonatal Intensive Care Units Audit Group. Mortality and adverse neurologic outcomes are greater in preterm male infants. Pediatrics 129, 124-131 (2012).

9. Tambe, P., Sammons, H. M. \& Choonara, I. Why do young children die in the UK? A comparison with Sweden. Arch. Dis. Child. archdischild-2014308059 (2015) doi:10.1136/archdischild-2014-308059.

10. Child Health Reviews UK, Clinical Outcome Review Programme. Overview of child deaths in the four UK countries.

https://www.rcpch.ac.uk/sites/default/files/CHR-UK_-_Retrospective_Epidemiological_Review_of_All-cause_Mortality_in_CYP.pdf (2013).

11. Ramnarayan, P., Craig, F., Petros, A. \& Pierce, C. Characteristics of deaths occurring in hospitalised children: changing trends. J. Med. Ethics 33, 255-260 (2007).

12. Muenchhoff, M. \& Goulder, P. J. R. Sex differences in pediatric infectious diseases. J. Infect. Dis. 209 Suppl 3, S120-126 (2014).

13. Amer, K., Roy, R. B. \& Nadel, S. Gender mortality differences of infants on PICU? A preliminary analysis. Arch. Dis. Child. 97, A36-A37 (2012).

14. Amer, K., Nadel, S. \& Basu-Roy, R. G57(P) Gender Mortality Differences of Infants on PICU? An Elaboration on Additional Analysis. Arch. Dis. Child. 98, A30-A30 (2013).

15. Paediatric Critical Care Minimum Data Set.

http://www.datadictionary.nhs.uk/data_dictionary/messages/supporting_data_sets/data_sets/paediatric_critical_care_minimum_data_set.asp? shownav=1.

16. The English Indices of Deprivation. https://www.gov.uk/government/uploads/system/uploads/attachment_data/file/6871/1871208.pdf (2010).

17. Payne, R. \& Abel, G. UK indices of multiple deprivation - a way to make comparisons across constituent countries easier. Health Serv. Q. Off. Natl. Stat. 53, (2012).

18. Slater, A., Shann, F., Pearson, G. \& Paediatric Index of Mortality (PIM) Study Group. PIM2: a revised version of the Paediatric Index of Mortality. Intensive Care Med. 29, 278-285 (2003).

19. Green, C. A. et al. Admission to hospital for bronchiolitis in England: trends over five decades, geographical variation and association with perinatal characteristics and subsequent asthma. Arch. Dis. Child. archdischild-2015-308723 (2015) doi:10.1136/archdischild-2015-308723.

20. Ghazaly, M. \& Nadel, S. Characteristics of children admitted to intensive care with acute bronchiolitis. Eur. J. Pediatr. 177, 913-920 (2018).

21. Discacciati, A., Oskarsson, V. \& Orsini, N. STPHCOXRCS: Stata module to check proportional-hazards assumption using restricted cubic splines. (2015).

22. Epstein, D. et al. Race/Ethnicity is not associated with mortality in the PICU. Pediatrics 127, e588-597 (2011).

23. Esteban, E., Bujaldon, E., Esparza, M., Jordan, I. \& Esteban, M. E. Sex differences in children with severe health conditions: Causes of admission and mortality in a Pediatric Intensive Care Unit. Am. J. Hum. Biol. Off. J. Hum. Biol. Counc. 27, 613-619 (2015).

24. Johansson Frigyesi, E., Andersson, P. \& Frigyesi, A. Boys have better short-term and long-term survival rates after intensive care admissions than girls. Acta Paediatr. Oslo Nor. 1992 106, 1973-1978 (2017).

25. Fraser, L. K. \& Parslow, R. Children with life-limiting conditions in paediatric intensive care units: a national cohort, data linkage study. Arch. Dis. Child. 103, 540-547 (2018).

26. Textor, J. \& Lis, M. Robust causal inference using directed acyclic graphs: the R package 'dagitty'. Int. J. Epidemiol. 45, 8 (2016).

27. Hughes, R. A., Heron, J., Sterne, J. A. C. \& Tilling, K. Accounting for missing data in statistical analyses: multiple imputation is not always the answer. Int. J. Epidemiol. 48, 1294-1304 (2019).

Page $7 / 11$ 
Table 1. Baseline demographics by sex

\begin{tabular}{|c|c|c|c|c|c|c|c|c|}
\hline & \multicolumn{4}{|c|}{ Female $(n=29,520)(41 \cdot 4 \%)$} & \multicolumn{4}{|c|}{ Male $(n=41,723)(58 \cdot 6 \%)$} \\
\hline & $\mathrm{n}^{*}$ & Mean or $\%$ & Median & IQR & $\mathrm{n}^{\star}$ & Mean or $\%$ & Median & IQR \\
\hline Age /days & 29,520 & $102 \cdot 51$ & $67 \cdot 00$ & $14-169$ & 41,723 & $98 \cdot 84$ & $60 \cdot 00$ & $13-161$ \\
\hline Length of stay /days & 29,514 & 5.66 & $2 \cdot 98$ & $1 \cdot 21-5 \cdot 96$ & 41,720 & $5 \cdot 71$ & $2 \cdot 96$ & $1 \cdot 18-5 \cdot 95$ \\
\hline Deprivation score & 25,105 & $27 \cdot 83$ & $24 \cdot 54$ & $13 \cdot 20-40 \cdot 10$ & 35,622 & $27 \cdot 45$ & $24 \cdot 24$ & $12 \cdot 86-39 \cdot 52$ \\
\hline PIM2R (2016) & 29,520 & 0.05 & $0 \cdot 02$ & $0 \cdot 01-0 \cdot 05$ & 41,723 & 0.05 & $0 \cdot 02$ & $0 \cdot 01-0 \cdot 05$ \\
\hline Duration of ventilation /days & 18,641 & 4.95 & $3 \cdot 00$ & & 26,552 & $5 \cdot 01$ & $3 \cdot 00$ & \\
\hline Ventilated during admission & 18,641 & $79 \cdot 28 \%$ & & & 26,552 & $77 \cdot 70 \%$ & & \\
\hline Planned admission & 29,485 & $40 \cdot 22 \%$ & & & 41,662 & $38 \cdot 01 \%$ & & \\
\hline Primary diagnosis bronchiolitis & 29,432 & $12 \cdot 44 \%$ & & & 41,625 & $12 \cdot 53 \%$ & & \\
\hline Primary diagnosis infection & 29,432 & $11 \cdot 37 \%$ & & & 41,625 & $11 \cdot 73 \%$ & & \\
\hline Inotropic support during admission & 29,519 & $24 \cdot 20 \%$ & & & 41,723 & $23 \cdot 53 \%$ & & \\
\hline Renal support during admission & 28,577 & $3 \cdot 53 \%$ & & & 40,254 & $3 \cdot 76 \%$ & & \\
\hline \multicolumn{9}{|l|}{ Gestation } \\
\hline 23-27 weeks & 1,782 & $7 \cdot 88 \%$ & & & 2,447 & $7 \cdot 59 \%$ & & \\
\hline 28-35 weeks & 4,297 & $19 \cdot 01 \%$ & & & 6,438 & $19 \cdot 96 \%$ & & \\
\hline $36-44$ & 16,522 & $73 \cdot 10 \%$ & & & 23,367 & $72 \cdot 45 \%$ & & \\
\hline Total & 22,601 & & & & 32,252 & & & \\
\hline \multicolumn{9}{|l|}{ Ethnicity } \\
\hline White & 17,741 & $76 \cdot 35 \%$ & & & 25,143 & $77 \cdot 10 \%$ & & \\
\hline Black & 1,297 & $5 \cdot 58 \%$ & & & 1,514 & $4 \cdot 64 \%$ & & \\
\hline Asian & 2,654 & $11 \cdot 42 \%$ & & & 3,796 & $11 \cdot 64 \%$ & & \\
\hline Mixed/Other & 1,543 & $6 \cdot 64 \%$ & & & 2,158 & $6 \cdot 62 \%$ & & \\
\hline Total & 23,235 & & & & 32,611 & & & \\
\hline
\end{tabular}

${ }^{*_{n}}$ is the number of cases with non-missing data. Where $\mathrm{n}$ is different to that in the main header, it indicates missing data; PIM2R (2016): Paediatric Index of Mortality version 2 revised and recalibrated in 2016

Table 1 (continued). Baseline demographics overall

\begin{tabular}{|c|c|c|c|c|c|}
\hline & & \multicolumn{4}{|c|}{ Total $(\mathrm{N}=71,243)$} \\
\hline & & $\mathrm{n}^{\star}$ & Mean or \% & Median & IQR \\
\hline Age /days & & 71,243 & $100 \cdot 36$ & 63.00 & $14-164$ \\
\hline Length of stay /days & & 71,234 & $5 \cdot 69$ & $2 \cdot 97$ & $1 \cdot 19-5 \cdot 96$ \\
\hline Deprivation score & & 60,727 & $27 \cdot 61$ & $24 \cdot 37$ & $13 \cdot 02-39 \cdot 75$ \\
\hline PIM2R (2016) & & 71,243 & 0.05 & 0.02 & $0 \cdot 01-0 \cdot 05$ \\
\hline Duration of ventilation /days & & 45,193 & 4.98 & $3 \cdot 00$ & \\
\hline Ventilated during admission & & 45,193 & $78 \cdot 35 \%$ & & \\
\hline Planned admission & & 71,147 & $38.92 \%$ & & \\
\hline Primary diagnosis bronchiolitis & & 71,057 & $12 \cdot 49 \%$ & & \\
\hline Primary diagnosis other infection & & 71,057 & $11 \cdot 58 \%$ & & \\
\hline Inotropic support during admission & & 71,242 & $23 \cdot 81 \%$ & & \\
\hline Renal support during admission & & 68,831 & $3 \cdot 67 \%$ & & \\
\hline \multicolumn{6}{|l|}{ Gestation } \\
\hline & 23-27 weeks & 4,229 & $7 \cdot 71 \%$ & & \\
\hline & 28-35 weeks & 10,735 & $19 \cdot 57 \%$ & & \\
\hline & $36-44$ & 39,889 & $72 \cdot 72 \%$ & & \\
\hline & Total & 54,853 & & & \\
\hline \multicolumn{6}{|l|}{ Ethnicity } \\
\hline & White & 42,884 & $76 \cdot 79 \%$ & & \\
\hline & Black & 2,811 & $5 \cdot 03 \%$ & & \\
\hline & Asian & 6,450 & $11 \cdot 55 \%$ & & \\
\hline & Mixed/Other & 3,701 & $6 \cdot 63 \%$ & & \\
\hline & Total & 55,846 & & & \\
\hline
\end{tabular}

$\star_{n}$ is the number of cases with non-missing data. Where $\mathrm{n}$ is different to that in the main header, it indicates missing data; PIM2R (2016): Paediatric Index of Mortality version 2 revised and recalibrated in 2016 
Table 2. Marginal probabilities of death within PICU and discharge from PICU for males and females at different time points

\begin{tabular}{llccccc}
\hline & & \multicolumn{4}{c}{ Time in days } \\
\cline { 3 - 6 } & & 2 & 5 & 10 & 20 & 40 \\
\hline Female & Dead & 0.018 & 0.028 & 0.034 & 0.040 & 0.045 \\
Male & Dead & 0.016 & 0.025 & 0.031 & 0.037 & 0.041 \\
Female & Discharged & 0.356 & 0.663 & 0.844 & 0.918 & 0.943 \\
Male & Discharged & 0.362 & 0.668 & 0.851 & 0.922 & 0.946 \\
\hline
\end{tabular}

Table 3. Parameters from the logistic regression model

\begin{tabular}{lccccc}
\hline & OR & SE & $\mathrm{P}$ & \multicolumn{2}{c}{$95 \% \mathrm{Cl}$} \\
\cline { 2 - 6 } Sex (male/female) & 0.860 & 0.033 & $<0.001$ & 0.797 & 0.927 \\
\hline PIM2R & 1.084 & 0.004 & $<0.001$ & 1.077 & 1.092 \\
\hline Constant & 0.039 & 0.003 & $<0.001$ & 0.033 & 0.046 \\
\hline
\end{tabular}

\section{Figures}

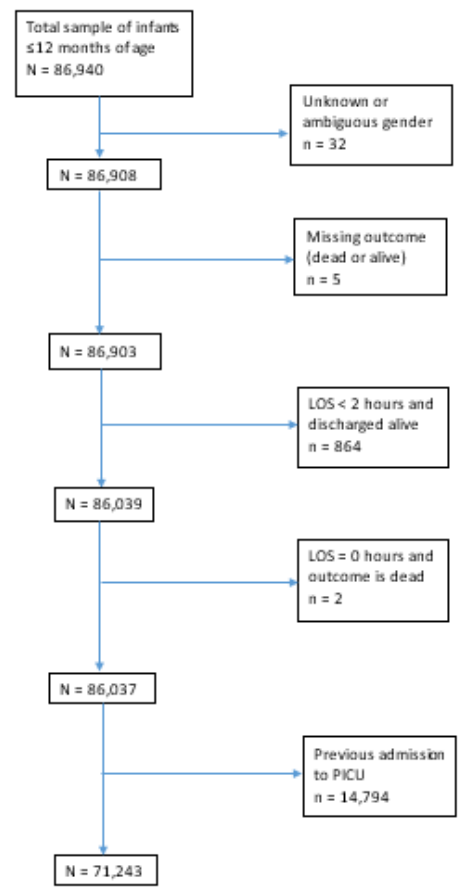

Figure 1

Data preparation 


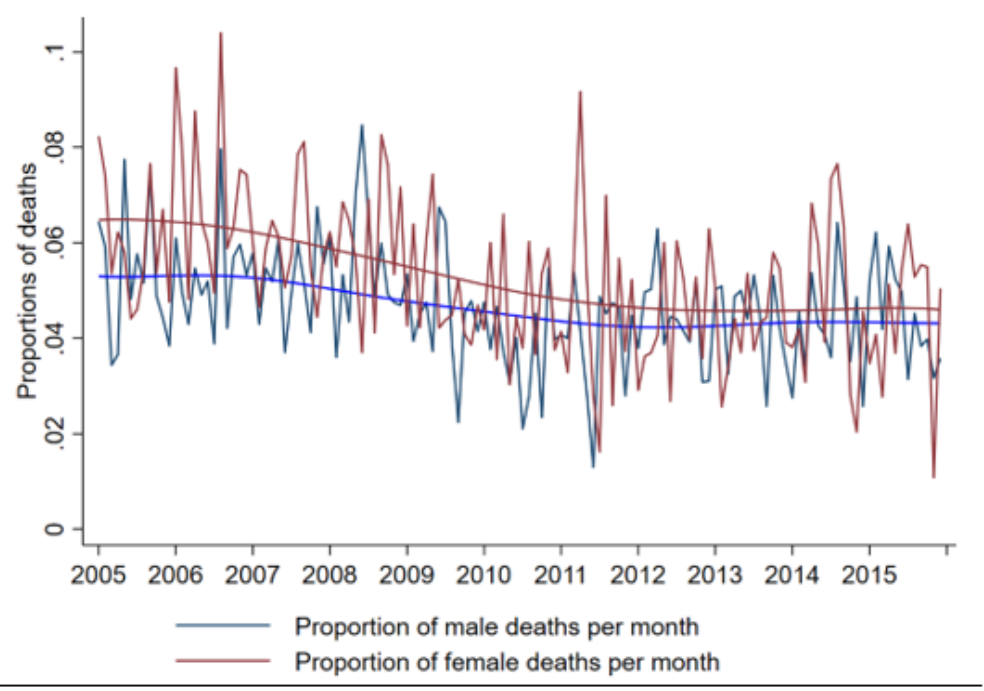

\section{Figure 2}

Time series plots of death for males and females The jagged lines are the variations in total deaths for males and females by month. The lowess smooth lines are the overall totals for average deaths per month for males and females over the 11-year study period, showing overall decline (smooth curves) in mortality.

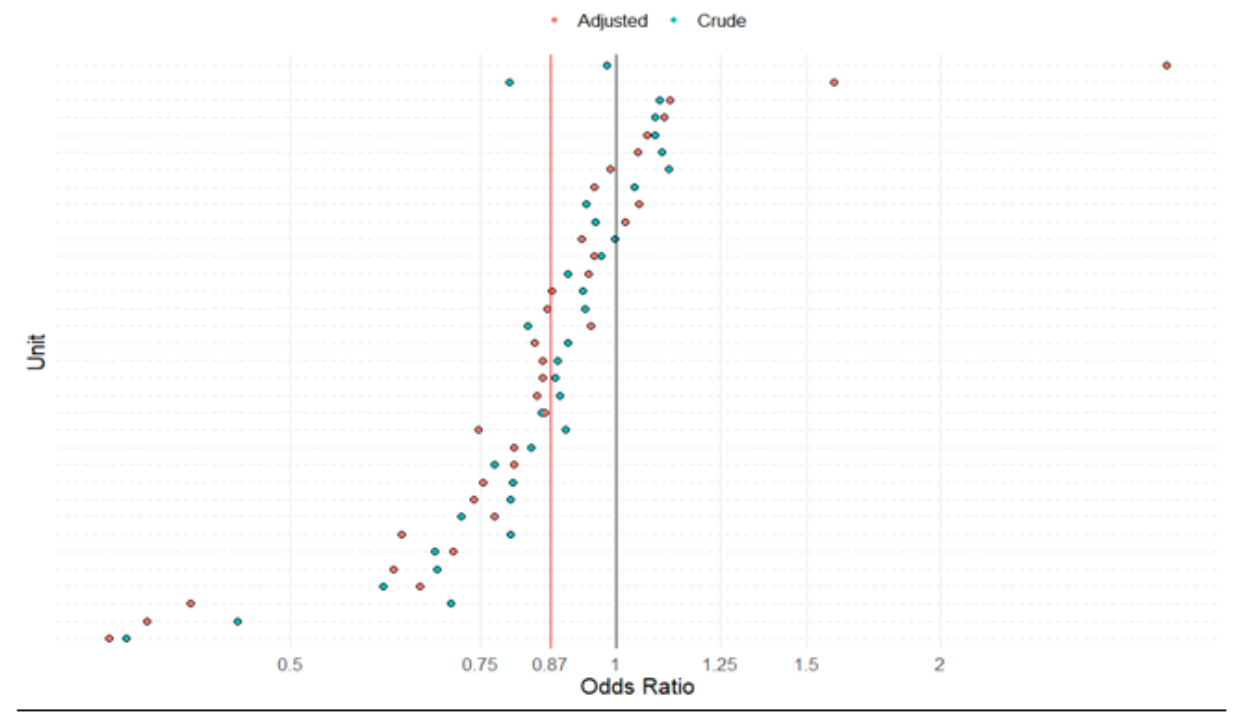

\section{Figure 3}

Crude and PIM2R adjusted mortality of males versus females for each critical care units The red vertical line is the overall PIM2R adjusted odds ratio from a cluster-robust model and the black vertical line is the null odds ratio value of one. The overall OR for male relative to female mortality is 0.87 $(95 \% \mathrm{Cl}, 0.81$ to $0.94, \mathrm{P}=0.001)$. Each dot represents individual units. OR $<1$ represents higher female mortality in that $\mathrm{PICU}, \mathrm{OR}>1$ represents higher male mortality. The PIM2R adjusted OR ensures that mortality comparison between the two sexes takes into account that the probability to be admitted in PICU depends on the severity of disease on admission. This plot can be viewed in conjunction with Table 3. 


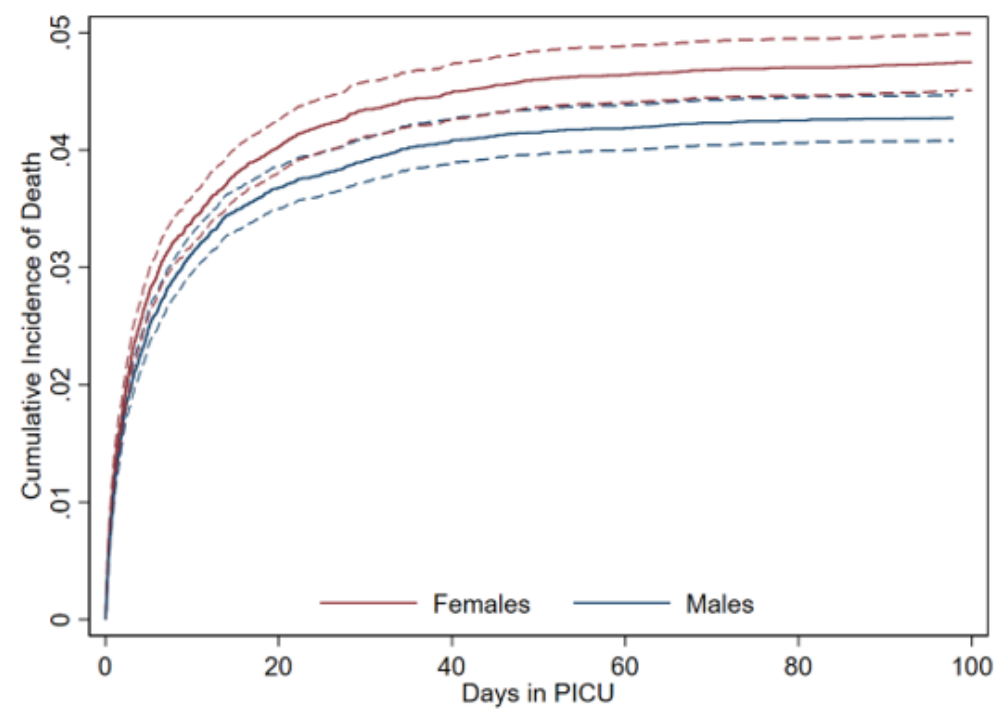

Figure 4

Non-parametric crude cumulative incidence of deaths in PICU Cause-specific cumulative incidence curves (CIF) for the outcome of death in PICU. The lines in the plot show the unadjusted cumulative incidence of death for males and females during the PICU stay. The dashed lines are the $95 \%$ confidence intervals. Clustering by units was not accounted for in this plot. Figure 4 can be viewed in conjunction with Table 2 , and support the finding of higher mortality for females relative to males. A test for equivalence of the CIF of death for females compared to males shows evidence of a higher risk for females (Log-rank Chi=4.91, $p=0.027$ ).

\section{Supplementary Files}

This is a list of supplementary files associated with this preprint. Click to download.

- SUPPLEMENTARYmaterials20200722.docx 